\title{
Age-dependent increased odds of cardiovascular risk factors in cancer survivors: Canadian Longitudinal Study on Aging cohort
}

\author{
A.A. Kirkham PhD, ${ }^{*}$ E. Pituskin PhD RN, ${ }^{\dagger}$ and S.E. Neil-Sztramko PhD ${ }^{\ddagger}$
}

\begin{abstract}
Background This study compared the odds of self-reported and objectively measured cardiovascular (CV) risk factors in a sample of Canadian cancer survivors and individuals without cancer.
\end{abstract}

Methods A nationally representative sample of 45 - to 85-year-old cancer survivors $(n=6288)$ in the Canadian Longitudinal Study on Aging were compared with individuals without cancer $(n=44,051)$.

Results The most prevalent risk factors in cancer survivors were all self-reported or easily measured in clinic: overweight or obesity (68.0\%), former smoking (62.9\%), fewer than 5 daily servings of fruits and vegetables (59.8\%), hypertension (43.7\%), and high waist circumference (47.0\%). After adjustment for sex and education, the odds ratios of several CV risk factors varied by age in cancer survivors and the non-cancer controls. At ages 50 and 60 , cancer survivors have increased odds of overweight or obesity, former smoking, hypertension, high waist circumference and truncal fat, diabetes, lung disease, and heart rate greater than $80 \mathrm{bpm}$ compared with non-cancer controls. At age 70, odds did not differ for many risk factors; at age 80, no differences were evident. Without modification by age, low physical activity was more prevalent in cancer survivors (odds ratio: 1.27; 95\% confidence interval: 1.17 to 1.39 ). There were no differences in the odds of $\mathrm{cv}$ risk factors measured by specialized equipment, including electrocardiography, carotid ultrasonography, spirometry, and dual-energy X-ray absorptiometry.

Conclusions The odds of several easy-to-assess CV disease risk factors are higher among middle-aged, but not older, cancer survivors relative to the general Canadian population. Initial assessment of $\mathrm{Cv}$ risk for middle-aged adults in the survivorship setting could be quickly and inexpensively performed using self-reported and easily measured metrics.

Key Words Survivorship, cardiovascular risk factors, hypertension, obesity, smoking, physical activity

Curr Oncol. 2020 August:27(4)e368-e376 www.current-oncology.com

\section{INTRODUCTION}

In 2017, 206,200 Canadians were expected to be diagnosed with cancer, and thanks to increasingly effective treatment, approximately 810,000 Canadians were living cancer survivors who had been diagnosed in the preceding 10 years ${ }^{1}$. Given the reduction in cancer mortality rates in recent decades, it has been recognized that individuals diagnosed with cancers having high survival rates (for example, breast, prostate, testicular, sarcoma) will often die from cardiovascular (CV) disease rather than from cancer $^{2-5}$. For example, diagnosis and treatment of breast cancer increases the risk of $\mathrm{CV}$ disease-related death by up to $50 \%$ relative to the risk for age-matched women without breast cancer ${ }^{6}$.

Given the significant risk of CV mortality after a cancer diagnosis and the growing number of cancer survivors, it is crucial to characterize CV morbidity in cancer survivors. Numerous modifiable risk factors-including hypertension, smoking, diabetes, physical inactivity, low fruit and vegetable consumption, and overweight or obesity-have been associated with an increased risk of $\mathrm{CV}$ disease in the general population ${ }^{7}$. Those risk factors can be easily assessed by self-report, but a number of objective 
measurements such as waist circumference, resting heart rate, electrocardiography, and carotid intima media thickness (IMT) have also been associated with cV risk ${ }^{8-10}$. However, the prevalence of the objective risk factors especially has not been well characterized in cancer-affected populations.

Identification of the risk factors that are the most common and that show increased odds in cancer survivors compared with the general population can help care providers to streamline risk assessment and can inform prevention, early detection, and management of CV disease in the survivorship setting. The purpose of the present analysis was to

describe the prevalence of self-reported and objectively measured cV disease risk factors in a nationally representative cohort of Canadian cancer survivors, and determine the odds ratio of those risk factors in cancer survivors relative to individuals without a history of cancer.

\section{METHODS}

The Canadian Longitudinal Study on Aging (CLSA) is an ongoing population-based cohort study of 51,338 adults between the ages of 45 and $85^{11}$. The CLSA excluded residents of institutions, the 3 territories, some remote regions, federal First Nations reserves, and other First Nations settlements; non-English or non-French speakers; fulltime Canadian Armed Forces members; and individuals with cognitive impairment. A “comprehensive cohort” of 30,097 participants (from among the 51,338 total) provided self-reported information about health and health determinants through in-home computer-assisted personal interviews; they also participated in objectively measured physical assessments through in-person assessments at 11 data collection sites. A "tracking cohort" of 21,241 participants (from among the 51,338 total) provided only self-reported health and health determinant information through computer-assisted telephone interviews; they did not participate in data collection. Data collection occurs every 3 years; to date, the initial baseline assessment is complete and forms the basis of the present analysis. The University of Alberta's Research Ethics Board 2 provided approval for the analysis.

\section{Study Sample}

"Cancer survivors" were defined as individuals who reported a history of cancer other than non-melanoma skin cancer. Participants reporting a history of only non-melanoma skin cancer were not included in the cancer survivor group because that diagnosis tends to reduce the sensitivity of self-reporting of a cancer history ${ }^{12}$ and has relatively less impact on long-term health. The CLSA did not collect information about the timing of a cancer diagnosis or the types of treatments received. Participants who did not provide valid responses to the questions used to identify cancer status or whose responses included confounders used in multivariate models were excluded from all analyses.

\section{Variables}

The CV risk factors included were those identified by the World Heart Federation ${ }^{7}$ and a number of primarily objectively measured variables that have also demonstrated prognostic value for CV disease. Table I provides details of measurement techniques and definitions. Objectively measured variables were obtained for the comprehensive cohort ( $n=30,097$ subset) only by trained CLSA staff at data collection sites. Detailed standard operating procedures are available at the CLSA Web site ${ }^{16}$.

\section{Analyses}

Analytical weights provided by the CLSA were applied to all analyses as recommended by the CLSA for representativeness ${ }^{17}$. Descriptive statistics were calculated as weighted means with standard deviation or as weighted percentages with $95 \%$ confidence intervals. Demographics were compared between the groups using the independent samples $t$-test for continuous variables and the chi-square test for categorical variables. The prevalences of CV risk factors were calculated as weighted percentages. Univariate logistic regression was used to compare the prevalences of CV risk factors between groups, and unadjusted odds ratios (ORs) are presented. Multivariable logistic regression was also performed to adjust for key confounders, including age, sex, and education level (as a surrogate for socioeconomic status). Ethnicity was also explored, but did not appear to confound the relationships examined, and so was not included. We also hypothesized that cancer might modify the age-related trajectory of risk factors for CV disease. To explore that possibility, we conducted our analyses using an age-by-cancer-status interaction term. When the addition of an interaction improved the model fit (determined by the Akaike information criterion), age-specific adjusted ORs (adjusted for sex and education) for representative ages of $50,60,70$, and 80 years are presented. When the addition of the interaction term did not improve model fit, oRs adjusted for age, sex, and education are presented. Analyses were conducted using the SAS software application (version 9.4: SAS Institute, Cary, NC, U.S.A.).

\section{RESULTS}

Of the 51,338 individuals total in the CLSA dataset, 999 (1.9\%) were excluded from the present analyses for invalid responses about their history of cancer or education. The analyses therefore included 50,339 individuals, of whom 6288 were cancer survivors (12.5\%). On average, cancer survivors had a similar body mass index, but were 6.4 years older than individuals without cancer (Table II). The most common types of cancer were breast (23.6\%) and prostate (18.1\%, Table II).

The most prevalent self-reported risk factors in cancer survivors were overweight or obesity (68.0\%), former smoking (62.9\%), fewer than 5 daily servings of fruits and vegetables $(59.8 \%)$, and hypertension $(43.7 \%$, Table III). The prevalence of measured overweight or obesity $(71.4 \%$, Table III) was similar to self-report. Otherwise, carotid IMT (58.9\%) and waist circumference $(47.0 \%)$ measurements exceeding known risk thresholds were the only objectively measured risk factors as prevalent as the self-reported risk factors (Table IV).

In comparing the odds of risk factors between cancer and non-cancer groups, a significant age-by-cancer-status interaction was evident both for self-reported and measured hypertension and for self-reported and measured 
TABLE I Techniques and definitions of cardiovascular risk factor measurement

\begin{tabular}{|c|c|c|}
\hline Risk factor & Assessment type & Definition \\
\hline Hypertension & Self-report & Any history \\
\hline $\begin{array}{l}\text { Uncontrolled } \\
\text { hypertension }\end{array}$ & $\begin{array}{l}\text { Average of } 2 \text { nd }-6 \text { th seated measurements } \\
\text { at } 60 \mathrm{~s} \text { intervals (BpTRU device }{ }^{\mathrm{a}} \text { ) }\end{array}$ & $\geq 140 / \geq 90 \mathrm{mmHg}$ \\
\hline Diabetes & Self-report & Any history \\
\hline Overweight or obesity & $\begin{array}{l}\text { Self-report and measured as BMI using } \\
\text { height in metres and weight in kilograms }\end{array}$ & $\mathrm{BMI} \geq 25.0 \mathrm{~kg} / \mathrm{m}^{2}$ \\
\hline $\begin{array}{l}\text { High-risk waist } \\
\text { circumference }^{10}\end{array}$ & $\begin{array}{l}\text { Measured at the level of the navel (men) } \\
\text { or narrowest part of the waist (women) }\end{array}$ & $>102 \mathrm{~cm}$ for men, $>88 \mathrm{~cm}$ for women \\
\hline Smoking status & Self-report & $\begin{array}{c}\text { Categorized as current smoker, former smoker, } \\
\text { or never-smoker }\end{array}$ \\
\hline $\begin{array}{l}\text { Servings of fruits and } \\
\text { vegetables daily }\end{array}$ & $\begin{array}{l}\text { Self-report SCREEN II }{ }^{13} \text { question: } \\
\text { In general, how many servings of fruits } \\
\text { and vegetables do you eat in a day? }\end{array}$ & Report of fewer than 5 servings daily \\
\hline Physical activity & $\begin{array}{l}\text { Self-report using the Physical Activity Scale } \\
\text { for the Elderly }{ }^{14}\end{array}$ & Lowest relative to highest quartile for sample \\
\hline Abnormal ECG & $\begin{array}{l}\text { Measured by } 12 \text {-lead electrocardiography } \\
\text { (MAC } 1600 \text { Resting ECG System }{ }^{\text {b }} \text { ) }\end{array}$ & Abnormalities of heart rate, rhythm, electrical conductance \\
\hline $\begin{array}{l}\text { High-risk carotid intima } \\
\text { media thickness }\end{array}$ & $\begin{array}{l}\text { Highest average of up to } 5 \text { measurements } \\
\text { on either side (Vivid i ultrasound system }{ }^{\text {b) }}\end{array}$ & $\geq 0.75 \mathrm{~mm}^{8}$ \\
\hline $\begin{array}{l}\text { High- and very-high-risk } \\
\text { resting heart rate }\end{array}$ & $\begin{array}{l}\text { Average of the 2nd-6th seated measurements } \\
\text { at } 60 \mathrm{~s} \text { intervals (BpTRU device }{ }^{\mathrm{a}} \text { ) }\end{array}$ & $>80$ bpm and $>90$ bpm respectively $^{9}$ \\
\hline Lung disease & Self-reported & $\begin{array}{l}\text { History of emphysema, chronic bronchitis, COPD, } \\
\text { or chronic changes in lungs attributable to smoking }\end{array}$ \\
\hline COPD & $\begin{array}{l}\text { Best of 3-8 measurements using an } \\
\text { Easy on-PC spirometer }{ }^{c}\end{array}$ & $\mathrm{FEV}_{1} / \mathrm{FVC}<0.70$ \\
\hline Trunk fat & Dual-energy X-ray absorptiometry & $\begin{array}{l}\text { Highest relative to lowest quartile of fat in trunk region } \\
\text { for sample }\end{array}$ \\
\hline Lean mass & Dual-energy X-ray absorptiometry & $\begin{array}{l}\text { Lowest relative to highest quartile of total lean mass } \\
\text { (kilograms/height in metres squared) from whole study sample }\end{array}$ \\
\hline Low grip strength & $\begin{array}{l}\text { Maximum of } 3 \text { trials on dominant hand } \\
\text { (Tracker Freedom grip dynamometer }{ }^{d} \text { ) }\end{array}$ & $<30 \mathrm{~kg}$ for men, $<20 \mathrm{~kg}$ for women \\
\hline Low gait speed & Timed $4 \mathrm{~m}$ walk test & $<0.8 \mathrm{~m} / \mathrm{s}$ \\
\hline Sarcopenia & $\begin{array}{l}\text { Dual-energy X-ray absorptiometry, grip } \\
\text { strength dynamometry, } 4 \mathrm{~m} \text { walk test }\end{array}$ & $\begin{array}{l}\text { European Working Group on Sarcopenia in Older Persons } \\
\text { criteria for low lean mass (appendicular lean mass/height }{ }^{2} \\
\leq 7.23 \mathrm{~kg} / \mathrm{m}^{2} \text { for men and } 5.67 \mathrm{~kg} / \mathrm{m}^{2} \text { for women) and gait } \\
\text { speed }<0.8 \mathrm{~m} / \mathrm{s} \text { or low grip strength as already defined }{ }^{15}\end{array}$ \\
\hline $\begin{array}{l}\text { Osteoporosis and } \\
\text { osteopenia }\end{array}$ & Dual-energy X-ray absorptiometry T score & -2.5 or less and less than -1.0 respectively \\
\hline \multicolumn{3}{|c|}{$\begin{array}{l}\text { VSM MedTech, Vancouver, BC. } \\
\text { General Electric, Toronto, ON. } \\
\text { ndd Medical Technologies, Andover, MA, U.S.A. } \\
\text { JTECH Medical, Midvale, UT, U.S.A. }\end{array}$} \\
\hline
\end{tabular}

overweight or obesity; for self-reported diabetes or lung disease, and current and former smoking status; and for measured waist circumference, resting heart rate, gait speed, and dual-energy X-ray absorptiometry-derived trunk fat and lean mass (Tables III and IV). For most of those variables, ORs were significantly higher for cancer survivors at age 50 , with stepwise reductions by decade, such that by age 80, no differences in ORs were evident between cancer survivors and individuals without cancer. The exception to that pattern was that the odds of lowest-quintile lean mass and low gait speed were higher in cancer survivors at older ages (Table IV). There was no evidence of age modification on the odds of poor diet and physical activity behaviours. The adjusted odds of cancer survivors being in the lowest quartile of physical activity was elevated (Table III). There was no difference in the adjusted odds for consuming fewer than 5 daily servings of fruits and vegetables (Table III). For several of the risk factors requiring specialized equipment (abnormal electrocardiogram, high-risk carotid IMT, measured chronic obstructive pulmonary disease, sarcopenia, low grip strength, osteopenia, and osteoporosis), the oRs were not modified by age, and the adjusted ons were nonsignificant, 
TABLE II Weighted characteristics for the study sample

\begin{tabular}{|c|c|c|c|c|c|}
\hline \multirow[t]{2}{*}{ Characteristic } & \multicolumn{2}{|c|}{ Cancer $(n=6,288)$} & \multicolumn{2}{|c|}{ No cancer $(n=44,051)$} & \multirow[t]{2}{*}{$p$ Value } \\
\hline & Weighted & $95 \% \mathrm{Cl}$ & Weighted & $95 \% \mathrm{CI}$ & \\
\hline Mean age (years) & 65.6 & 65.3 to 65.8 & 59.2 & 59.1 to 59.3 & $<0.001$ \\
\hline Mean BMI ${ }^{\mathrm{a}}$ (self-report in $\mathrm{kg} / \mathrm{m}^{2}$ ) & 27.8 & 27.7 to 28.0 & 27.7 & 27.7 to 27.8 & 0.19 \\
\hline Mean $\mathrm{BMI}^{\mathrm{b}}$ (measured in $\mathrm{kg} / \mathrm{m}^{2}$ ) & 28.3 & 28.1 to 28.4 & 28.0 & 27.9 to 28.0 & $<0.01$ \\
\hline Female sex (\%) & 56.0 & 54.6 to 57.3 & 50.7 & 50.2 to 51.2 & $<0.001$ \\
\hline \multicolumn{6}{|l|}{ Marital status ${ }^{\mathrm{a}}(\%)$} \\
\hline Single (never married) & 7.1 & 6.5 to 7.9 & 7.9 & 7.6 to 8.1 & $<0.001$ \\
\hline Married or common-law & 70.4 & 69.0 to 71.4 & 75.7 & 75.2 to 75.9 & \\
\hline Divorced, separated, or widowed & 22.5 & 21.4 to 23.6 & 16.5 & 16.1 to 16.8 & \\
\hline Education (\%) & & & & & $<0.001$ \\
\hline Less than secondary school & 8.3 & 7.6 to 9.1 & 5.7 & 5.5 to 5.9 & \\
\hline Secondary school diploma & 11.7 & 10.9 to 12.7 & 10.6 & 10.4 to 10.9 & \\
\hline Some postsecondary & 8.2 & 7.4 to 8.9 & 7.0 & 6.8 to 7.3 & \\
\hline Postsecondary degree or diploma & 71.8 & 70.5 to 72.9 & 76.7 & 76.2 to 77.0 & \\
\hline Ethnicity (\%) & & & & & $<0.001$ \\
\hline White & 93.4 & 92.7 to 94.1 & 90.7 & 90.4 to 91.1 & \\
\hline Asian & 1.1 & 0.8 to 1.4 & 2.6 & 2.4 to 2.8 & \\
\hline Indigenous & 3.2 & 2.7 to 3.8 & 3.8 & 3.6 to 4.0 & \\
\hline Black & 0.8 & 0.6 to 1.1 & 1.0 & 0.9 to 1.1 & \\
\hline Other & 1.4 & 1.1 to 1.7 & 1.9 & 1.7 to 2.0 & \\
\hline Cancer type ${ }^{\mathrm{C}}(\%)$ & & & & & - \\
\hline Breast & 23.6 & 22.4 to 24.7 & & & \\
\hline Prostate & 18.1 & 17.1 to 19.1 & & & \\
\hline Melanoma & 14.3 & 13.4 to 15.3 & & & \\
\hline Colon or rectum & 10.5 & 9.6 to 11.4 & & & \\
\hline Female genital organs & 9.3 & 8.6 to 10.1 & & & \\
\hline Bladder & 3.8 & 3.3 to 4.2 & & & \\
\hline Non-Hodgkin lymphoma & 3.8 & 2.8 to 3.8 & & & \\
\hline Ovary & 3.2 & 2.7 to 3.6 & & & \\
\hline Thyroid & 3.1 & 2.6 to 3.6 & & & \\
\hline Lung & 2.9 & 2.5 to 3.3 & & & \\
\hline Kidney & 2.8 & 2.4 to 3.2 & & & \\
\hline Lymphoid or hematopoietic & 2.6 & 2.2 to 3.1 & & & \\
\hline Lip, oral cavity, or pharynx & 2.1 & 1.7 to 2.5 & & & \\
\hline Leukemia & 2.0 & 1.6 to 2.4 & & & \\
\hline Digestive organs & 2.0 & 1.6 to 2.3 & & & \\
\hline Other $^{d}$ & 6.1 & 5.5 to 6.8 & & & \\
\hline
\end{tabular}

a Missing data: BMI self-report $(n=241)$ and marital status $(n=14)$.

b Measured in 29,266 participants.

c Includes multiple cancer types for $12 \%$ of participants reporting more than 1 cancer type.

d Cancers of the eye, brain, central nervous system, mesothelium, soft tissue, male genital organs, bone, articular cartilage, respiratory organs, intrathoracic organs, pancreas, endocrine system, and urinary tract, plus "don't know" and unspecified.

$\mathrm{BMI}=$ body mass index.

suggesting that there is no difference between cancer survivors and individuals without cancer (Table IV).

\section{DISCUSSION}

The coexistence of cancer and CV disease is a major public health concern that is likely to continue to increase in prevalence as the population age ${ }^{18}$. Various factors associated with CV risk or health can be present and interact before a cancer diagnosis, during the active treatment period, and in the post-treatment survivorship setting ${ }^{19}$. Furthermore, numerous types of cancer treatment are associated with direct CV toxicity (for example, anthracycline chemotherapy, thoracic radiation, therapies targeting the human epidermal growth receptor, tyrosine kinase inhibitors, and immunotherapies), and the resulting manifestations are highly variable: left ventricular dysfunction, heart failure, cardiac structural or electrical abnormalities, hypertension, vascular thrombosis, and ischemia, among others $^{20-23}$. The resulting patient-level CV morbidity is 


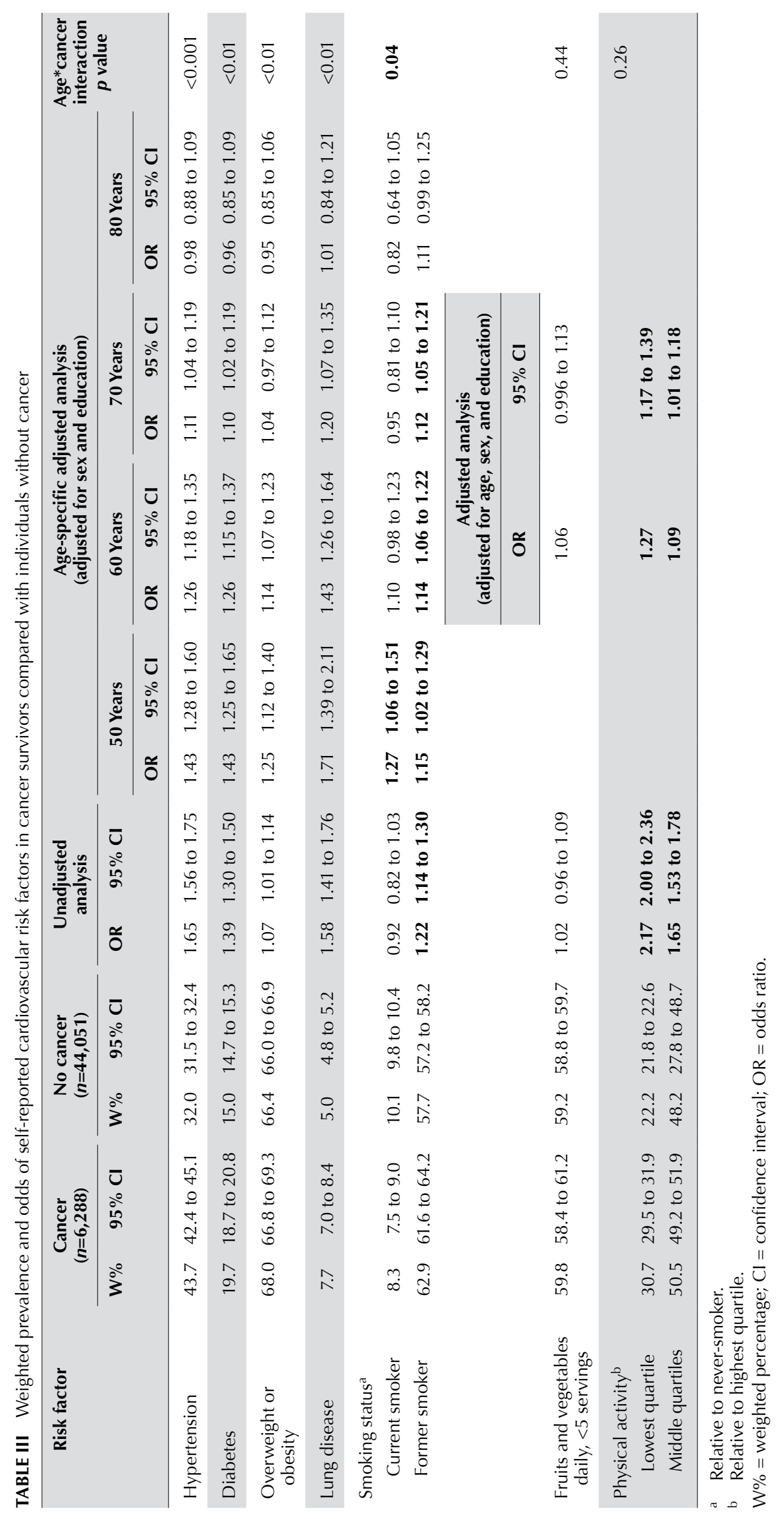




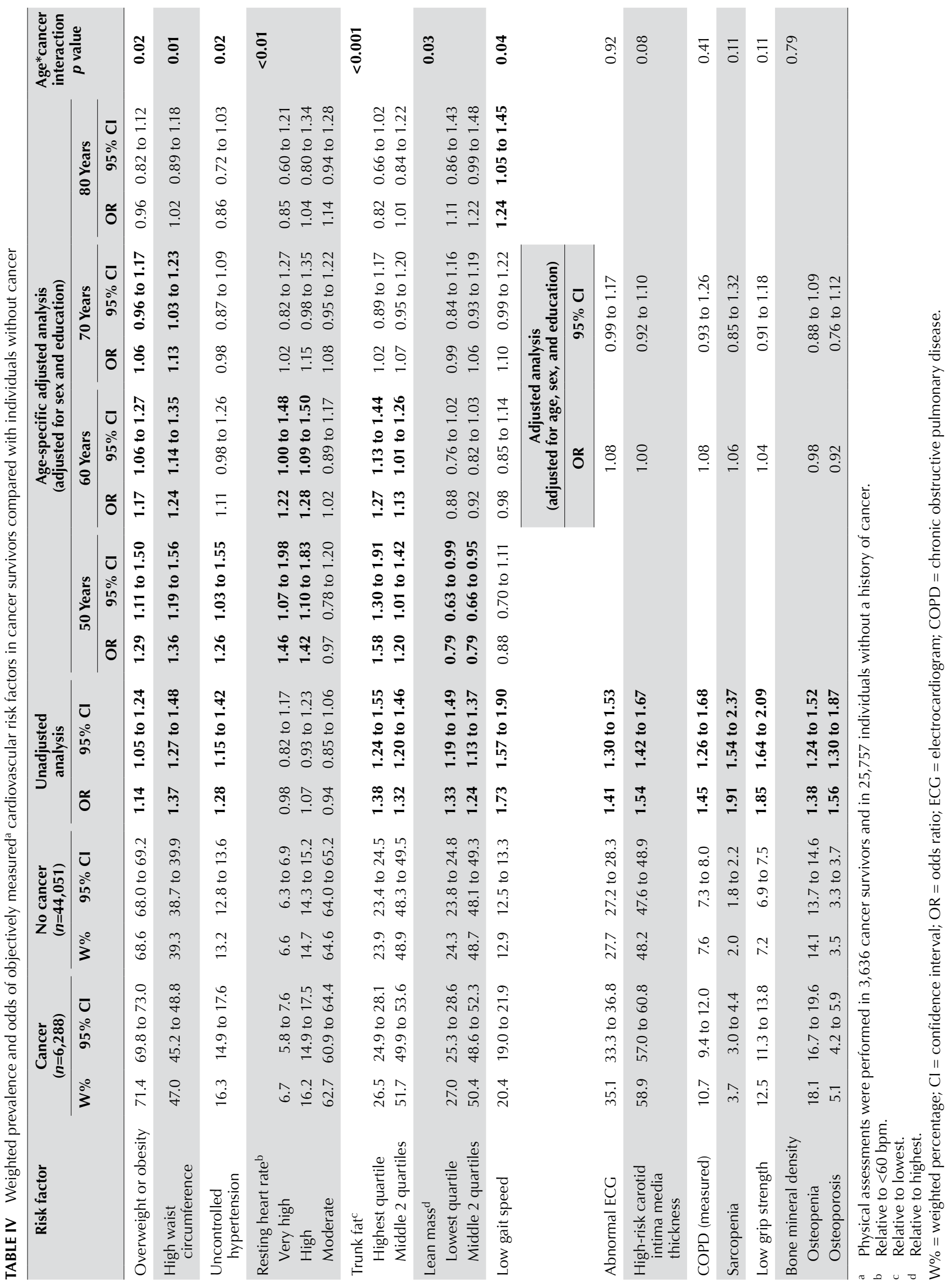


therefore likely to manifest as a highly variable, complex, multifactorial disease process.

Characterization of the resulting CV morbidity in cancer survivors is required to inform prevention, early detection, and management of $c$ health in the survivorship setting. In the present study, we described the prevalence of a broad suite of self-reported and objectively measured CV risk factors in a representative sample of Canadians with a history of cancer, and we compared the odds of those risk factor prevalences with the odds in a group of individuals without cancer. Importantly, our results have actionable implications for clinicians engaged in survivorship care. One of the primary findings was that the cV risk factors that are the most prevalent in cancer survivors are self-reported or easily measured in clinic, thus lowering the barrier to detection. A second overarching finding was that, in our 45- to 85-year-old cohort, the odds for most of the CV risk factors studied are increased in younger, but not older, cancer survivors, thus highlighting a target population potentially requiring heightened monitoring for CV risk.

Many of the traditional modifiable risk factors identified by the World Heart Federation are related to lifestyle behaviours. A cancer diagnosis and subsequent treatment is associated with the potential for direct CV toxicity as well as for lifestyle toxicity ${ }^{19}$. "Lifestyle toxicity" refers to a worsening of lifestyle behaviours after a cancer diagnosis and treatment; it can include reductions in physical activity or diet quality, and increased body weight and psychological stress ${ }^{19}$. In the present analysis, nearly all of the lifestyle-related CV risk factors were elevated in cancer survivors, reinforcing the suggestion that lifestyle toxicity is a prevalent issue at any point after diagnosis-not only during active treatment, but also in the post-treatment survivorship period ${ }^{19}$. For example, consumption of fewer than 5 daily servings of fruits and vegetables was an extremely prevalent CV risk factor: approximately $60 \%$ in the CLSA cohort, including among cancer survivors. That risk factor is a long-established threshold associated with improved CV and general health ${ }^{24}$ and thus represents an easily accessible target for CV risk management in the cancer survivorship setting. The odds of being in the lowest quartile of physical activity was $27 \%$ greater for cancer survivors than for individuals without cancer. Analysis of another Canadian dataset found that only cancer survivors who identified as currently having cancer, and not those with a past cancer status, were less likely to be active than were age-matched adults ${ }^{25}$. Timing of treatment might be an important effect modifier that warrants further attention. Risk factors closely related to diet and physical activity (overweight or obesity, and high waist circumference) were elevated in cancer survivors at representative ages of 50 and 60 years. Lastly, smoking as a single lifestyle behaviour is an extremely important target for CV risk in all populations, because it is thought to be independently responsible for $10 \%$ of all cases of $\mathrm{CV}$ disease in the general population ${ }^{26}$. Although $62.9 \%$ of the cancer survivors in our cohort reported being former smokers, smoking cessation is an actionable and important target for $\mathrm{CV}$ risk management in the $8.3 \%$ who report current-smoker status.

Self-reported hypertension and diabetes were the CV risk factors with the highest ons (both 1.43). The "silent killer," hypertension, is also either undiagnosed or uncontrolled in $16 \%$ of cancer survivors. Diagnosis and management of hypertension is an aspect of CV risk management that should and could be easily improved in cancer survivors through increased awareness at all phases of the cancer trajectory. That finding has important health implications, because antihypertensive therapy has been shown to reduce the incidence of stroke by $35 \%-40 \%$, myocardial infarction by $20 \%-25 \%$, and heart failure by more than $50 \%{ }^{27}$. Lifestyle modification is also an indispensable aspect of blood pressure management, because it can lower blood pressure and also enhance drug efficacy ${ }^{27}$. A combination of 2 or more of the following recommendations will achieve optimum results: 10-pound weight loss, increased consumption of fruits and vegetables, consumption of less than 2.4 g sodium per day, brisk walking for 30 minutes daily, and limitation of alcohol consumption to 2 drinks daily for men and 1 drink daily for women ${ }^{27}$.

The $20 \%$ prevalence of diabetes among cancer survivors in the present analysis is also a significant cause for concern. Diabetes significantly increases the risk of death from coronary artery disease by $50 \%$, peripheral vascular disease by $20 \%-30 \%$, and stroke by $10 \%{ }^{28}$. A healthy lifestyle is also important for the prevention and management of diabetes ${ }^{28}$. Results from the present study clearly identify the importance of diagnosis and treatment of hypertension and diabetes, including enhancement of healthy lifestyle behaviours, as a CV risk management technique in cancer survivors.

We also assessed a number of nontraditional CV risk factors to identify whether additional important assessment tools are available to monitor CV risk in cancer survivors. We observed no differences in the adjusted odds of measured risk factors requiring specialized equipment (electrocardiogram; carotid IMT; and measured chronic obstructive pulmonary disease, sarcopenia, and low grip strength) between cancer survivors and individuals without a history of cancer. For cancer survivors at specific ages, odds were increased for risk factors requiring simple measurements, including resting heart rate (ages 50 and 60) and low gait speed (from a $4 \mathrm{~m}$ walk test, age 80 ). The odds for dual energy X-ray absorptiometry-derived trunk fat and for waist circumference were both increased at ages 50 and 60 , but the latter assessment, being simpler and less costly, is suggested for use in clinical practice. That finding can be used to save costs, resources, and time, at least in the initial assessment or screening for CV risk in cancer survivors. Of course, the assessments requiring specialized equipment, with their increased costs, will still provide valuable diagnostic information if the patient's history or preliminary assessment suggests a need for further investigation.

Based on the study results, we suggest that, for clinicians providing cancer survivorship care, assessment of CV risk for individuals less than 70 years of age should particularly focus on a careful medical and lifestyle behaviour history; assessment of resting heart rate, blood pressure, body mass index, and waist circumference; and careful screening for risk of diabetes, in addition to other standard risk assessments as indicated. Treatment plans for survivors should focus on lifestyle behaviour (diet, exercise, smoking) counselling or referrals, and antihypertensive medications for those with confirmed hypertension. 
The present analysis is limited by the cross-sectional nature of the data and the inability to perform subgroup analyses for cancer type and treatment type. It is likely that CV risk factor prevalences were underestimated by survivorship bias. Individuals with the greatest CV risk might have been less likely to be included in the CLSA representative sample because of poor health or mortality. A strength of the analysis was the use of specialized measurementsincluding electrocardiography, carotid ultrasonography, spirometry, and dual-energy X-ray absorptiometry-for approximately $60 \%$ of the participants in the cohort. Those data represent a unique characteristic compared with prior publications that have exclusively used self-reported CV risk factors in larger cohorts.

\section{CONCLUSIONS}

The CV risk factors that are most prevalent in cancer survivors or that have elevated odds in cancer survivors compared with individuals without a history of cancer are selfreported or easily measured in clinic. In the 45 - to 85 -yearold nationally representative CLSA cohort, the CV risk was greatest in younger cancer survivors. Our findings can be used in the survivorship setting to guide an accessible and time-efficient preliminary CV risk assessment. An initial assessment of CV risk for middle-aged cancer survivors should consist of self-report of smoking status; daily servings of fruits and vegetables; physical activity; and personal history of diabetes, hypertension, and lung disease. Basic in-clinic measurements should consist of height and weight to assess overweight or obesity status, resting blood pressure and heart rate, and waist circumference. Diagnosis and appropriate management of hypertension and diabetes should receive particular attention. Treatment plans for the management of $\mathrm{CV}$ risk in cancer survivors should consider lifestyle (diet, exercise, smoking) behaviour counselling or referrals as indicated, and antihypertensive medications for those with confirmed hypertension.

\section{ACKNOWLEDGMENTS}

This research was made possible using the data and biospecimens collected by the CLSA. Funding for the CLSA is provided by the Government of Canada through the Canadian Institutes of Health Research (grant reference: LSA 9447) and the Canada Foundation for Innovation. This research was conducted using the CLSA dataset (Baseline Tracking, version 3.3, and Baseline Comprehensive, version 3.2, under application no. 170310). The CLSA is led by Drs. Parminder Raina, Christina Wolfson, and Susan Kirkland. The opinions expressed in this manuscript are those of the authors and do not reflect the views of the CLSA. AAK is supported by Postdoctoral Fellowships from the Canadian Institutes of Health Research, Alberta Innovates Health Solutions, and Susan G. Komen. SENS is supported by a Postdoctoral Fellowship from the Canadian Institutes of Health Research.

\section{CONFLICT OF INTEREST DISCLOSURES}

We have read and understood Current Oncology's policy on disclosing conflicts of interest, and we declare that we have none.

\section{AUTHOR AFFILIATIONS}

*Current: Faculty of Kinesiology and Physical Education, University of Toronto, Toronto, ON; former: Department of Biomedical Engineering, University of Alberta, Edmonton, AB; ${ }^{\dagger}$ Faculty of
Nursing, University of Alberta, Edmonton, AB; ${ }^{\ddagger}$ McMaster University, Hamilton, ON.

\section{REFERENCES}

1. Canadian Cancer Society's Advisory Committee on Cancer Statistics. Canadian Cancer Statistics 2017. Toronto, ON: Canadian Cancer Society; 2017.

2. Youn P, Milano MT, Constine LS, Travis LB. Long-term cause-specific mortality in survivors of adolescent and young adult bone and soft tissue sarcoma: a population-based study of 28,844 patients. Cancer 2014;120:2334-42.

3. Shikanov S, Kocherginsky M, Shalhav AL, Eggener SE. Cause-specific mortality following radical prostatectomy. Prostate Cancer Prostatic Dis 2012;15:106-10.

4. Fosså SD, Gilbert E, Dores GM, et al. Noncancer causes of death in survivors of testicular cancer.J Natl Cancer Inst 2007; 99:533-44.

5. Gernaat SAM, Ho PJ, Rijnberg N, et al. Risk of death from cardiovascular disease following breast cancer: a systematic review. Breast Cancer Res Treat 2017;164:537-55.

6. Riihimaki M, Thomsen H, Brandt A, Sundquist J, Hemminki K. Deathcausesinbreastcancerpatients.Ann Oncol2012;23:604-10.

7. World Heart Federation. Cardiovascular Disease Risk Factors. Geneva, Switzerland: World Heart Federation; 2013.

8. Aminbakhsh A, Mancini GB. Carotid intima-media thickness measurements: what defines an abnormality? A systematic review. Clin Invest Med 1999;22:149-57.

9. Zhang D, Shen X, Qi X. Resting heart rate and all-cause and cardiovascular mortality in the general population: a metaanalysis. CMAJ 2016;188:E53-63.

10. Riebe D, Ehrman JK, Liguori G, Magal M, eds. ACSM's Guidelines for Exercise Testing and Prescription. 10th ed. Philadelphia, PA: Wolters Kluwer; 2018.

11. Raina PS, Wolfson C, Kirkland SA, et al. The Canadian Longitudinal Study on Aging (CLSA). Can J Aging 2009;28:221-9.

12. Earle $\mathrm{C}$ on behalf of the Ontario Institute for Cancer Research. Sensitivity and specificity of self-reported cancer history compared to cancer registry [abstract 234]. J Clin Oncol 2017;35:. [Available online at: https://ascopubs.org/doi/ abs/10.1200/JCO.2017.35.8_suppl.234; cited 14 July 2020]

13. Reimer HD, Keller HH, Maitland SB, Jackson J. Nutrition screening index for older adults (SCREEN II) demonstrates sex and age invariance. J Nutr Elder 2010;29:192-210.

14. Granger CL, Parry SM, Denehy L. The self-reported Physical Activity Scale for the Elderly (PASE) is a valid and clinically applicable measure in lung cancer. Support Care Cancer 2015;23:3211-18.

15. Dam TT, Peters KW, Fragala M, et al. An evidence-based comparison of operational criteria for the presence of sarcopenia. J Gerontol A Biol Sci Med Sci 2014;69:584-90.

16. Canadian Longitudinal Study on Aging (CLSA). Physical Assessments [Web page]. Hamilton, ON: CLSA; 2017. [Available at: https://www.clsa-elcv.ca/researchers/physical-assessments; cited 18 February 2019]

17. Raina P, Kirkland S, Wolfson C. Sampling and Computation of Response Rates and Sample Weights for the Tracking (Telephone Interview) Participants and Comprehensive Participants. Hamilton, ON: Canadian Longitudinal Study on Aging; 2017. [Available online at: https://www.clsa-elcv.ca/doc/1041; cited 18 February 2019]

18. Harrison J, Friese C, Barton D, Janz N, Pressler S, Davis M. Heart failure and long-term survival among older women with breast cancer. Oncol Nurs Forum 2018;45:A1-11.

19. Kirkham AA, Beaudry R, Paterson DI, Mackey JR, Haykowsky MJ. Curing breast cancer and killing the heart: a novel model to explain elevated cardiovascular disease and mortality risk among women with early stage breast cancer. Prog Cardiovasc Dis 2019;62:116-26. 
20. Force T, Kolaja KL. Cardiotoxicity of kinase inhibitors: the prediction and translation of preclinical models to clinical outcomes. Nat Rev Drug Disc 2011;10:111-26.

21. Pai VB, Nahata MC. Cardiotoxicity of chemotherapeutic agents: incidence, treatment and prevention. Drug Saf 2000; 22:263-302.

22. Ewer MS, Gibbs HR, Swafford J, Benjamin RS. Cardiotoxicity in patients receiving trastuzumab (Herceptin): primary toxicity, synergistic or sequential stress, or surveillance artifact? Semin Oncol 1999;26(suppl 12):96-101.

23. Curigliano G, Cardinale D, Dent S, et al. Cardiotoxicity of anticancer treatments: epidemiology, detection, and management. CA Cancer J Clin 2016;66:309-25.

24. Steffen LM. Eat your fruit and vegetables. Lancet 2006;367: 278-9.
25. Neil SE, Gotay CC, Campbell KL. Physical activity levels of cancer survivors in Canada: findings from the Canadian Community Health Survey. J Cancer Surviv 2014;8:143-9.

26. Mendis S, Puska P, Norrving B, eds. Global Atlas on Cardiovascular Disease Prevention and Control. Geneva, Switzerland: World Health Organization; 2011.

27. United States, Department of Health and Human Services, National Institutes of Health. Seventh Report of the Joint National Committee on Prevention, Detection, Evaluation, and Treatment of High Blood Pressure. Bethesda, MD: National Heart, Lung, and Blood Institute; 2004.

28. Narayan KM, Gregg EW, Fagot-Campagna A, Engelgau MM, Vinicor F. Diabetes - a common, growing, serious, costly, and potentially preventable public health problem. Diabetes Res Clin Pract 2000;50 (suppl 2):S77-84. 